\title{
ESTRESSE OCUPACIONAL EM ENFERMEIROS DE UM HOSPITAL UNIVERSITÁRIO*
}

\author{
Taciana Mirella Batista dos Santos ${ }^{1}$, Iracema da Silva Frazão², Delmilena Maria Aquino Ferreira ${ }^{3}$
}

\begin{abstract}
RESUMO: Estudo comparativo tece como objetivo investigar o estresse entre enfermeiros e os fatores relacionados. A população da pesquisa foi formada por 44 enfermeiros que atuavam em unidades hospitalares. Os dados socioeconômicos e profissionais e os fatores de fontes de estresse foram apresentados sob forma de Escala de Likert, e coletados por meio de instrumento auto-aplicável e analisados com auxílio do programa estatístico Epi-Info 2004 versão 3.2 e Statistical Package for the Social Sciences para Windows. Os resultados foram agrupados em cinco categorias: Conflito de funções, Gerenciamento de pessoal, Sobrecarga de trabalho, Situações críticas e Relacionamento interpessoal. Os enfermeiros atuantes no setor aberto apresentaram maior escore de estresse, sendo a sobrecarga de trabalho o principal fator associado. O conhecimento e discussão sobre fatores de estresse podem contribuir para a busca de melhores condições de trabalho e interferir na qualidade de vida e desempenho profissional do enfermeiro.
\end{abstract}

PALAVRAS-CHAVE: Esgotamento profissional; Trabalho; Enfermagem.

\section{WORKLOAD-RELATED STRESS IN NURSES IN A UNIVERSITY HOSPITAL*}

\begin{abstract}
A comparative study that investigates stress among nurses and the factors related to it. The research population was 44 nurses working in hospital units. The socio-economic and professional data and the factors regarded as the sources of stress were presented using a Likert Scale. They were collected using a self-reporting tool and analyzed with the help of the statistical program Epi-Info 2004 version 3.2 and Statistical Package for the Social Sciences for Windows. The results were grouped in five categories: job conflict, staff management, overwork, critical situations and interpersonal relationships. The nurses working in the open sector presented the highest stress levels, overwork being the principal factor related with this. Awareness and discussion of stress factors can contribute to the improvement of nursing professionals' working conditions, quality of life and professional performance.
\end{abstract}

KEYWORDS: Bornout, professional; Work; Nursing.

\section{ESTRÉS OCUPACIONAL EN ENFERMEROS DE UN HOSPITAL UNIVERSITARIO*}

RESUMEN: Estudio comparativo que tiene como objetivo investigar el estrés entre enfermeros y los factores asosiados. La población de la investigación fue compuesta por 44 enfermeros que trabajaban en unidades hospitalares. Los datos socioeconómicos y profesionales y los factores de fuentes de estrés fueron presentados en forma de Escala de Likert, y recogidos por medio de instrumento autoaplicable y analizados con ayuda del programa estadístico Epi-Info 2004 versión 3.2 y Statistical Package for the Social Sciences para Windows. Los resultados fueron agrupados en cinco categorías: Conflicto de funciones, Administración de personas, Sobrecarga de trabajo, Situaciones críticas y Relaciones interpersonales. Los enfermeros que trabajaban en sector abierto presentaron mayor índice de estrés, siendo la sobrecarga de trabajo el principal factor asociado. El conocimiento y discusión sobre factores de estrés pueden contribuir para la búsqueda de mejores condiciones de trabajo e interferir en la cualidad de vida y desempeño profesional del enfermero.

PALABRAS CLAVE: Agotamiento professional; Trabajo; Enfermería.

\footnotetext{
*Artigo baseado na monografia do curso de Graduação em Enfermagem apresentada em 2008 à Universidade Federal de PernambucoUFPE.

${ }^{1}$ Enfermeira Residente de Nefrologia no Hospital das Clínicas de Pernambuco da UFPE.

${ }^{2}$ Enfermeira. Doutora em Serviço Social. Professora do Curso de Enfermagem e do Programa de Pós-Graduação em Enfermagem da UFPE. Líder do Grupo de Pesquisa Saúde Mental e Qualidade de Vida no Ciclo Vital UFPE.

${ }^{3}$ Enfermeira. Especializada do Curso de Enfermagem do Trabalho da Faculdade dos Guararapes/PE.
}

Autor correspondente:

Taciana Mirella Batista dos Santos

Universidade Federal de Pernambuco

R. Padre Nestor de Alencar, 7342 - 54450-220 - Jaboatão dos Guararapes-PE-Brasil

E-mail: tacianamirella@hotmail.com

Recebido: $16 / 05 / 10$ Aprovado: 17/02/11 


\section{INTRODUÇÃO}

As primeiras referências à palavra estresse têm por sinônimos "aflição" e "adversidade" e datam do século XIV, sendo seu emprego assistemático. No século XVII, o vocábulo, que tem origem no latim stringere, passou a ser usado para designar opressão, desconforto e adversidade ${ }^{(1)}$. Posteriormente, em 1936, o termo estresse foi designado como uma síndrome produzida por vários agentes nocivos, com ênfase na resposta não específica do organismo a situações que o enfraquecessem ou que o fizessem adoecer; foi designado síndrome geral de adaptação ou síndrome do stress biológico ${ }^{(2)}$.

$\mathrm{O}$ estresse, em pequeno grau, é necessário ao organismo, pois colabora com o bom desempenho das funções orgânicas e psíquicas; o nível positivo de estresse é denominado eustresse. Porém, quando ocorrem situações prejudiciais ao cotidiano do indivíduo, as situações que seriam benéficas e comuns deixam de afetá-lo positivamente, e passam a afetá-lo negativamente, desenvolvendo quadro patológico. Esse processo negativo, caracterizado por situações aflitivas, é denominado distresse ${ }^{(2)}$.

Os riscos para a saúde, relacionados com o trabalho, dependem do tipo de atividade profissional e das condições em que é desempenhada. Os serviços de saúde, e de um modo particular os hospitais, proporcionam aos funcionários condições de trabatho reconhecidamente insalubres. Os enfermeiros encontram-se expostos, do ponto de vista etiológico, a fatores de risco de natureza física, química, biológica e psicossocial - tais fatores se fazem sentir com grande intensidade e justificam a inclusão da profissão de enfermagem no grupo das profissões desgastantes ${ }^{(3)}$.

O trabalho em turnos é uma característica do exercício profissional da Enfermagem, caracterizado por assistência ininterrupta ${ }^{(4)}$, e em períodos utilizados por outros trabalhadores para dormir, descansar, usufruir do lazer e do convívio social e familiar.

$O$ excesso de trabalho, tanto em termos quantitativos como qualitativos, é uma fonte frequente de estresse. Por sobrecarga quantitativa, entende-se o excesso de atividades a realizar num determinado período de tempo; a qualitativa refere-se a excessivas exigências em relação às competências, conhecimentos e habilidades do trabalhador ${ }^{(5)}$.

Assim, esta pesquisa teve por objetivo investigar o estresse entre enfermeiros e os fatores relacionados a enfermeiros lotados em unidade hospitalar.

\section{METODOLOGIA}

Esta pesquisa caracteriza-se por ser de caráter exploratório, descritivo e comparativo e de natureza quantitativa. Foi realizada no Hospital de Clínicas de Pernambuco, localizado na Cidade Universitária do Recife. Para a seleção da amostra de sujeitos foram inicialmente selecionadas todas as unidades fechadas e, em número proporcional e de forma aleatória as unidades abertas, totalizando dez unidades. As unidades fechadas foram: Terapia Intensiva Geral e de Recuperação de Cirurgia Cardíaca; Terapia Intensiva Neonatal; Central de Material Esterilizado; Bloco Cirúrgico e Hemodiálise. As unidades aberas foram: Cirúrgica; Pediátrica; Transplante; Traumatoortopedia e Alojamento Conjunto.

A população do estudo foi formada pela totalidade de enfermeiros atuantes nas unidades do estudo de abril a maio de 2008 e que concordaram em participar e responderar ao instrumento de coleta autoaplicável, totalizando 44 enfermeiros.

$\mathrm{O}$ instrumento de coleta de dados constituiu de um questionário autoaplicável, que foi entregue aos enfermeiros, e por eles respondido. Esse instrumento foi composto por duas partes: a primeira, de autoria das pesquisadoras, composto por 17 perguntas relativas a informações pessoais, profissionais e hábitos de vida. A segunda parte constou de 49 perguntas relativas aos Fatores e Fontes de Estresse. Esse instrumento foi traduzido e validado no Brasil por Lautert em $1999^{(6)}$. As afirmativas do segundo instrumento foram apresentadas sob a forma de Escala de Likert, a qual permite discriminar diferentes pontos de vista.

Os dados coletados foram digitados em uma planilha do programa estatístico Epi-Info 2004 versão 3.2. Este mesmo programa foi utilizado para o cálculo dos dados sociodemográficos do estudo. Para os demais cálculos, utilizou-se o programa Statistical Package for the Social Sciences para Windows.

A análise referente aos fatores e fontes de estresse foi agrupada de acordo com a semelhança semântica. Este procedimento prende-se ao fato de que itens individuais são menos fidedignos do que os testes inteiros $^{(7)}$.

O estudo foi aprovado pelo Comitê de Ética da Universidade Federal de Pernambuco (n. 069/2008).

\section{RESULTADOS}

A amostra foi composta por $93,2 \%$ de mulheres 
e $6,82 \%$ do sexo masculino, sendo $56,82 \%$ casados, $2,27 \%$ com união estável, e $31,82 \%$ solteiros; $47,73 \%$ não possuíam filhos; a faixa etária prevalente foi de 36 a 40 anos, relativa a $25 \%$ dos participantes.

Foram encontradas cargas horárias de trabalho entre 30 e 40 horas semanais, e também ultrapassando 70 horas de trabalho semanal; a maioria $(34,09 \%)$ trabalha entre 40 e 50 horas semanais (Tabela 1).

Tabela 1 - Carga horária semanal de enfermeiros. Recife, 2008

\begin{tabular}{lcccc}
\hline Carga horária & \multicolumn{3}{c}{ Setor aberto } & \multicolumn{3}{c}{ Setor fechado } \\
& N & \% & N & \% \\
\hline 40 a 50 & 7 & 15,91 & 8 & 18,18 \\
51 a 60 & 5 & 11,36 & 4 & 9,09 \\
61 a 70 & 3 & 6,82 & 4 & 9,09 \\
Mais de 70 & 2 & 4,55 & 2 & 4,55 \\
Não informou & 2 & 4,55 & 7 & 15,91 \\
\hline
\end{tabular}

Quanto aos fatores de estresse identificados, cinco categorias foram agrupadas conforme apresentadas a seguir: Conflito de Funções; Sobrecarga de Trabalho; Relacionamento Interpessoal; Gerenciamento de Pessoal; e Situações Críticas.

Conforme a figura 1, entre os enfermeiros que trabalham de 40 a 50 horas no setor aberto, encontramos maior escore na variável Sobrecarga de Trabalho $(3,09)$, enquanto que no setor fechado, no Gerenciamento de Pessoal $(3,16)$ e Conflito de Funções $(3,10)$.

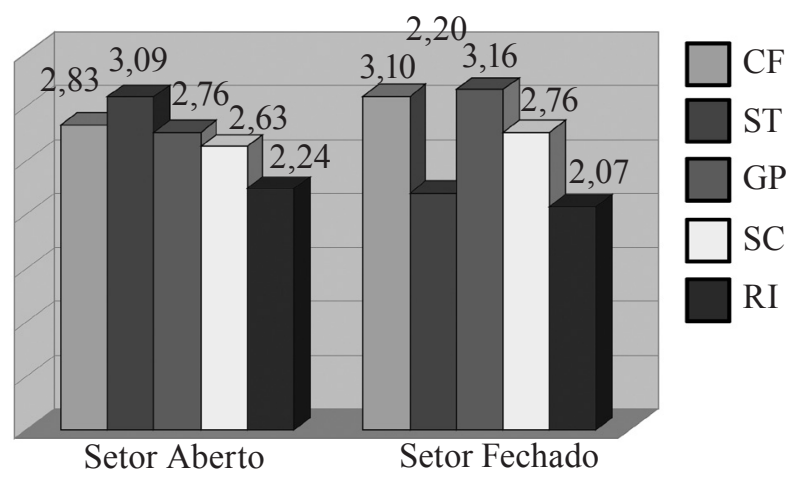

Figura 1 - Distribuição dos escores de estresse e correlação dos fatores e fontes de estresse com a carga horária semanal de trabalho de 40 a 50 horas entre enfermeiros. Recife, 2008

Legenda: CF - Conflito de Funções; ST - Sobrecarga de Trabalho; GP - Gerenciamento de Pessoal; SC - Situações Críticas; RI Relacionamento Interpessoal

Entre os que trabalham entre 51 e 60 horas semanais, o maior escore de estresse no setor aberto foi referente ao Conflito de Funções (3,09); por outro lado, o setor fechado tem o Gerenciamento de Pessoal, seguido pela Sobrecarga de Trabalho os maiores escores. O escore de estresse para situações críticas não foi discrepante para os setores, conforme apresentado na figura 2.

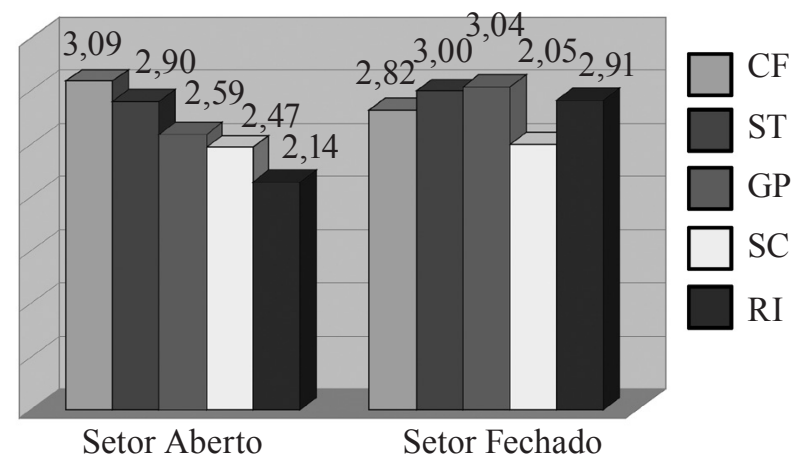

Figura 2 - Distribuição dos escores de estresse e correlação dos fatores e fontes de estresse com a carga horária semanal de trabalho de 51 a 60 horas entre enfermeiros. Recife, 2008

Legenda: CF - Conflito de Funções; ST - Sobrecarga de Trabalho; GP - Gerenciamento de Pessoal; SC - Situações Críticas; RI Relacionamento Interpessoal

Para quem trabalha entre 61 e 70 horas semanais (Figura 3), encontramos que o setor aberto apresentou maiores escores de estresse para todas as categorias.

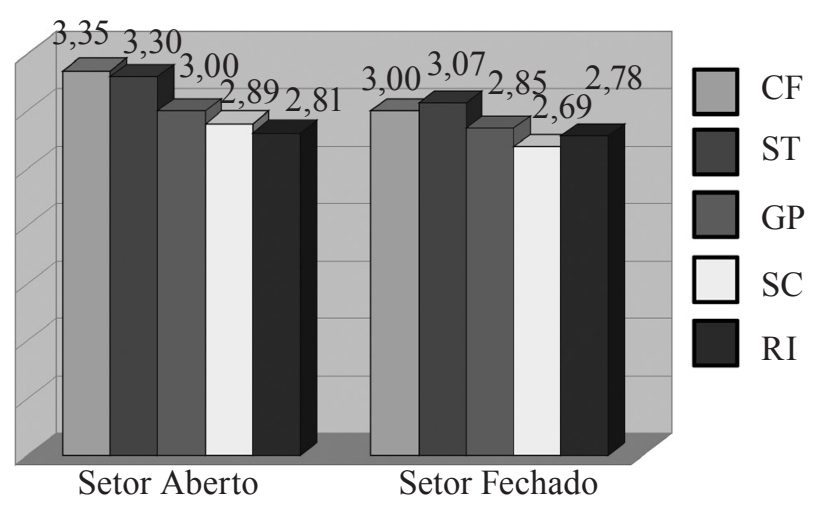

Figura 3 - Distribuição dos escores de estresse e correlação dos fatores e fontes de estresse com a carga horária semanal de trabalho de 61 a 70 horas entre enfermeiros. Recife, 2008

Legenda: CF - Conflito de Funções; ST - Sobrecarga de Trabalho; GP - Gerenciamento de Pessoal; SC - Situações Críticas; RI Relacionamento Interpessoal

Já a figura 4 mostra que os enfermeiros que trabalham mais de 70 horas semanais em setor aberto apresentaram maiores escores de estresse para todas as funções, em relação ao setor fechado. 


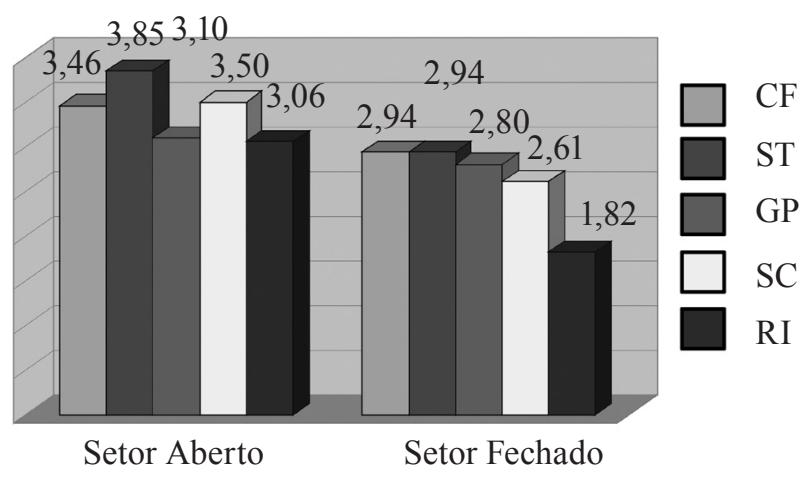

Figura 4 - Distribuição dos escores de estresse e correlação dos fatores e fontes de estresse com a carga horária semanal de trabalho acima de 70 horas entre enfermeiros. Recife, 2008

Legenda: CF - Conflito de Funções; ST - Sobrecarga de Trabalho; GP - Gerenciamento de Pessoal; SC - Situações Críticas; RI Relacionamento Interpessoal

\section{DISCUSSÃO}

Os entrevistados, em sua maioria, eram mulheres. Como um reflexo do modelo profissional de Enfermagem instituído no país, o corpo profissional da enfermagem brasileira continua, em geral, sendo constituído por mulheres, tanto em seus níveis de qualificação universitária quanto nos níveis médio e técnico. Entretanto, o ingresso masculino na profissão mantém-se estável, com $10 \%$ das vagas de ensino ocupadas por homens ${ }^{(8)}$. A estruturação deste cenário de predominância feminina na profissão deve-se, em parte, ao modelo de Enfermagem implantado no Brasil, no século XX, no qual o ingresso à profissão esteve aberto apenas às mulheres ${ }^{(9)}$. $\mathrm{O}$ número de enfermeiros sem filhos retrata a realidade mundial. Hoje, percebemos que as mulheres são mães cada vez mais tarde, porque colocam os estudos e a carreira no topo das suas prioridades, e só depois vem a ideia do casamento e, eventualmente, a vinda de filhos, sempre com a opção de não ter filhos e, mesmo quando os têm, isso não as ocupa o suficiente para abandonar a carreira $^{(10)}$.

A pesquisa mostrou que os enfermeiros, de maneira geral, se encontram estressados, sendo a carga horária de trabalho uma das causas que representa maior desgaste físico e mental. Considerando que o tempo destinado ao lazer é inversamente proporcional ao tempo destinado ao trabalho.

A maioria dos entrevistados respondeu que trabalha mais de 50 horas semanais, o que significa dizer que trabalha em outros serviços. Isto porque, na instituição onde foi realizada a pesquisa, a carga horária máxima permitida é de 40 horas semanais. Sabe-se que, frequentemente, a dupla jornada de trabalho se faz necessária pela situação econômica dos trabalhadores na Enfermagem. Os salários baixos levam o indivíduo a procurar outras fontes de renda e, com isso, ele passa a assumir múltiplas funções, o que pode levar a frustração, cansaço e desvalorização profissional, entre outros ${ }^{(11)}$. A carga horária elevada está entre as principais causas de acidentes; a necessidade de mais um emprego exige do profissional de Enfermagem a permanência da maioria dos anos produtivos em ambiente insalubre, o que aumenta o tempo de exposição aos riscos ocupacionais ${ }^{(12)}$.

A sobrecarga de trabalho no setor aberto o obteve maior escore da pesquisa. Observamos que, para Conflito de Funções no setor aberto, os escores aumentam na medida em que aumenta a carga horária. Enfermeiros de setores abertos lidam diretamente com o público. Em pesquisa feita anteriormente, os enfermeiros relataram que a maior fonte de estresse foi o atendimento ao público ${ }^{(10)}$. Além disso, os enfermeiros de setores abertos estão em maior contato com outros profissionais de saúde e há maior número de pacientes para cada enfermeiro. Trabalhos anteriores relatam que excessiva demanda de pacientes, a sobrecarga de trabalho e o excesso de exigências são fatores predisponentes ao estresse $\mathrm{e}^{(13-14)}$.

Os enfermeiros lotados em unidades fechadas apresentaram menor escore de estresse no geral e no Relacionamento Interpessoal escore mínimo $(1,82)$, o menor da pesquisa. Esse resultado vai ao encontro de outros achados onde os enfermeiros consideram os desentendimentos com os companheiros de trabalho um estressor, principalmente em setor fechado, onde o convívio é mais intenso ${ }^{(13-14)}$.

O Relacionamento Interpessoal alcançou o menor escore de estresse para todas as cargas horárias, em relação às outras variáveis. Contudo, evidenciamos, nesta situação, que os escores de estresse apresentados foram diretamente proporcionais à carga horária dos enfermeiros. O relacionamento, mais ou menos harmonioso no trabalho, exige da gerência competências para resolver diferenças, utilizar ferramentas úteis. Elas visam o fortalecimento de fatores facilitadores que levam à construção coletiva de equipe. $\mathrm{O}$ trabalho na área da saúde requer dos enfermeiros atuação cooperativa, percepção criativa, visão positiva de conflitos, entre outras habilidades, para gerir 
interrelações de forma competente e sincronizada, em busca de objetivos comuns. Saber negociar depende de cada indivíduo, pois há fatores que podem influenciar, como cultura, crenças, valores, costumes, grau de conhecimento do problema, sentimentos, entre outros, contudo esse processo deve ser conduzido de forma amigável, independente do problema/conflito, e o resultado da negociação deve ser satisfatório para ambas as partes. Baseado nestes pressupostos, faz-se necessário que o profissional enfermeiro adquira conhecimentos para gerenciar essas situações conflituosas e desenvolva habilidades em realizar negociações ${ }^{(15)}$.

Convêm destacar que nas relações estabelecidas entre a equipe de enfermagem e dela com os pacientes e seus familiares deve haver o predomínio das seguintes características: presença de cooperação, cumplicidade, solidariedade, comprometimento profissional, além de uma comunicação efetiva, que possa resultar e determinar um ambiente de trabalho favorável à prestação do cuidado terapêutico ${ }^{(16)}$.

\section{CONCLUSÃo}

O estresse na atividade do enfermeiro está relacionado a diversas variáveis e ao próprio indivíduo. Porém, consideramos que não é fácil identificar onde se encontra o nó crítico. Verificamos que, embora os enfermeiros de unidades abertas apresentem maior escore de estresse em relação aos de unidades fechadas, não houve grande disparidade de resultados.

Não é difícil encontrar enfermeiros que atuam em mais de um hospital para suprir a renda familiar. Dessa maneira, o enfermeiro torna sua carga horária de trabalho extensa, comprometendo seu lazer e laços afetivos. Isso gera um desgaste físico e uma péssima qualidade de vida, que com certeza irá influenciar em sua atuação profissional, devido às condições em que exerce sua profissão.

Tem se falado muito da redução da carga horária para a Enfermagem. No entanto, como já foi dito, os enfermeiros mantêm uma extensa jornada de trabalho com intuito de melhorar a renda familiar. É preciso repensar as políticas públicas empregadas na área da saúde. Para valorização da profissão e melhor remuneração, cabe ao enfermeiro buscar pela qualificação profissional, para que possa atenuar o estresse sofrido pelos longos períodos de trabalho.

Portanto, é imprescindível estudar as causas do estresse do enfermeiro, para então prevenir e enfrentar os casos. Consideramos também de extrema importância avaliar cada situação para consolidar ações que se traduzam em melhoria das condições de trabalho do enfermeiro.

\section{REFERÊNCIAS}

1. Lipp M. Pesquisas sobre stress no Brasil: ocupações e grupos de risco. Campinas: Papirus; 1996.

2. Selye H. Stress and diseases. Science. 1955;122(3171):625-31.

3. Gaspar PJS. Enfermagem: profissão de risco e desgaste. Nursing. 1997;109(3):23-4.

4. De Martino MMF. Estudo da variabilidade circadiana da temperatura oral, ciclo vigília-sono e testes psicofisiológicos em enfermeiros de diferentes turnos de trabalho [tese]. Campinas (SP): Universidade Estadual de Campinas; 1996.

5. Peiró JM. Desencadenantes del estrés laboral. Madrid: Eudema; 1993.

6. Lautert L, Chaves BHE, Moura SSMG. O estresse na atividade gerencial do enfermeiro. Revista Panam Salud Publica. 1999;6(6):415-25.

7. Kerlinger FN. Metodologia da pesquisa em ciência sociais. São Paulo: EPU;1999.

8. Lopes MJ, Leal SMC. A feminização persistente na qualificação profissional da enfermagem brasileira. Cad Pagu. 2005;(24):105-25.

9. Pereira PF. Homens na enfermagem: atravessamentos de gênero na escolha, formação e exercício profissional [dissertação]. Porto Alegre (RS): Universidade Federal do Rio Grande do Sul; 2008.

10. Salvador AL. Monoparentalidade no feminino. Afreudite. 2010; Ano VI(11/12):25-30.

11. Fontana RT, Siqueira KI. O trabalho do enfermeiro em saúde coletiva e o estresse: análise de uma realidade. Cogitare Enferm.2009;14(3):491-8.

12. Simão SAF, Souza V, Borges RAA, Soares CRG, Cortez EA. Fatores associados aos acidentes biológicos entre profissionais de enfermagem. Cogitare Enferm. 2010;15(1):87-91.

13. Abranches SS, Marziale MHP. A situação ergonômica 
do trabalho de enfermagem em unidade básica de saúde [tese]. São Paulo (SP): Universidade de São Paulo; 2005.

14. Martins LMM, Bronzatti JAG, Vieira CSCA, Parra SHB, Silva YB. Agentes estressores no trabalho e sugestões para amenizá-los: opiniões de enfermeiros de pós-graduação. Rev Esc Enferm USP. 2000;34(1):52-8.

15. Corradini EM, Zgoda LTRW, Paul MFB. O gerenciamento de conflitos entre a equipe de enfermagem. Cogitare Enferm. 2008;13(2):184-93.

16. Wagner LR, Thofehm MB, Amestoy SC, Porto AR, Arrieira ICO. Relações interpessoais no trabalho: percepção de técnicos e auxiliares de enfermagem. Cogitare Enferm. 2009;14(1):107-13. 